\title{
A APLICAÇÃO DO CONTROLE ESTATÍSTICO DE PROCESSO NUMA INDÚSTRIA DE BENEFICIAMENTO DE CAMARÃO MARINHO NO ESTADO DO RIO GRANDE DO NORTE
}

\section{THE APPLICATION OF THE STATISTICAL CONTROL OF PROCESS IN AN INDUSTRY OF IMPROVEMENT OF MARINE SHRIMP IN THE STATE OF RIO GRANDE DO NORTE}

\author{
Itamar França da Silva Júnior ${ }^{1}$; Verônica Carneiro de Oliveira ${ }^{2}$ \\ ${ }^{1}$ Mestrando em Engenharia de Produção - PEP/UFRN, juniorufrn@yahoo.com.br \\ ${ }^{2}$ Mestrando em Engenharia de Produção - PEP/UFRN, veronicaoliveira@yahoo.com.br
}

Recebido para publicação em: 27/03/2005

Aceito para publicação em: 18/07/2005

\begin{abstract}
Resumo
Este artigo apresenta a aplicação do controle estatístico de processo numa indústria de processamento de camarão marinho no estado do Rio Grande do Norte, sendo aplicada para medir a quantidade de metabissulfito de sódio, conservante utilizado na maioria das indústrias pesqueiras, durante o processo de despesca e processamento do camarão. A variação de quantidade de metabisulfito de sódio utilizada no processo foi medida, e analisada através do uso de gráficos de controle. O resultado encontrado mostrou que o processo não se encontrava sob controle estatístico, logo, depois de descoberta e eliminada a causa determinável da variação o gráfico foi refeito e pode ser observado que o processo encontrou-se sob controle estatístico.
\end{abstract}

Palavras-chave: Qualidade, Controle estatístico de processo, Metabissulfito de sódio

\section{Introdução}

Nas últimas décadas, pode-se observar que os consumidores apresentam um perfil bem mais seletivo e exigente, gerando assim uma maior competitividade entre as empresas de diferentes setores, levando-as a procurarem suas vantagens competitivas principalmente em qualidade. Segundo Juran (1993), algo fundamental para a obtenção dessas vantagens competitivas é a melhoria dos processos produtivos.

De acordo com Thompson (1992), a concepção comum sobre controle de qualidade é que, isso é alcançado por uma grande investigação, uma boa atitude e trabalho duro. Ainda existem 
muitas companhias onde os empregados exibem todos esses atributos, mas a qualidade do produto é pobre.

Na segunda metade dos anos 20 foi desenvolvida, pelo Dr. Walter A. Shewhart, uma teoria de controle estatístico de qualidade. Para isso foram analisados diferentes processos e concluiu-se que todos eles apresentaram variação. Shewhart identificou nestas mudanças dois componentes, um estável, que parecia ser inerente ao processo, e um intermitente. Ele atribui variação inerente (aleatória) as causas acidentais e as causas indetermináveis, que não podiam ser economicamente descobertas e removidas sem que fossem feitas mudanças no processo e, a variação intermitente, a causas determináveis, que podiam ser observadas e eliminadas com um programa de diagnóstico.

A variação de qualquer característica de qualidade pode ser quantificada pela amostragem do resultado do processo e pela estimativa dos parâmetros da sua distribuição estatística. Mudanças na distribuição podem ser relevadas pelo gráfico destes parâmetros no tempo. As amostras geralmente consistem em mais de uma medição individual, e por isso são chamadas de "subgrupos". Os gráficos de variáveis são geralmente baseados em um subgrupo de 4 a 10 indivíduos, os gráficos de atributos num mínimo de 50 e, com freqüência, várias centenas de indivíduos (JURAN, 1993).

O Controle Estatístico de Processo (CEP) é uma técnica estatística desenvolvida para medir e analisar a variabilidade dos processos. Através do uso de gráficos de controle, pode-se detectar os defeitos, prevenir ajustes desnecessários no processo, estabelecer um diagnóstico e permitir o cálculo da capacidade do processo.

Com base nisso o presente estudo tem o objetivo de verificar o comportamento médio das análises de metabissulfito de sódio para cada sub-lote despescado de uma determinada fazenda de uma empresa de exportação de camarão no estado do Rio Grande do Norte. Esses resultados servirão para observar o comportamento das variações de $\mathrm{SO}_{2}$ no processo e provocar uma observação contínua no ato da despesca do camarão, já que a utilização deste conservante se dá no ato desta fase.

\section{Metodologia}

Para que o objetivo do trabalho seja alcançado, uma etapa metodológica deve ser cumprida, na qual as técnicas e ferramentas estatísticas são vistas como poderosas armas à disposição da indústria, baseadas na coleta, análise e interpretação de dados utilizados nas atividades de melhoria e controle da qualidade de produtos e serviços (SIQUEIRA, 1997). Tal fato justifica o desenvolvimento desse trabalho, pois se espera que a utilização dos gráficos de controle possa monitorar o processo em relação a utilização do metabissulfito de sódio. 
Uma análise exploratória será realizada a primeiro momento para que se conheça o comportamento da variável, posteriormente será aplicado os gráficos de controle, pois os mesmos, segundo Juran (1993), constituem uma ferramenta extremamente útil na identificação das variações envolvidas no processo.

\section{Gráficos de controle}

O gráfico de controle foi originalmente proposto em 1924, por W. A. Shewhart, que trabalhou no Bell Telephone Laboratories, com a intenção de eliminar variações anormais em um determinado processo produtivo com base em amostras periodicamente coletadas do processo. Eles são registros dos dados mensurados em um ponto crítico do processo, construídos num sistema de coordenadas cartesianas.

Segundo Vieira (1999), o gráfico de controle típico exibe três linhas paralelas: a central, que representa o valor médio do característico de qualidade; a superior, que representa o limite superiro de controle (LSC); e a inferior, que representa o limite inferior de controle (LIC).

No processo de construção desses limites comumente são usados em geral \pm 3 desvios padrões, que pode ser justificado pelos bons resultados obtidos na prática, com o nível de confiança estabelecido de 99,73\% na análise dos dados. Os pontos representam amostras tomadas em momentos diferentes. É usual unir os pontos por segmentos de reta, para melhor visualizar a evolução do característico de qualidade ao longo do tempo.

Os gráficos de controle devem ser implementados para monitorar as variáveis que influenciam a qualidade dos itens produzidos e também para se obter um estado de controle estatístico. Dessa forma, uma determinada variável de qualidade ou característica de qualidade é monitorada por meio de sucessivas amostras espaçadas no tempo. Estes fatos tornam o gráfico de controle uma das ferramentas mais utilizadas do Controle Estatístico de Processo (CEP), que possui ferramentas para monitorar o estado do processo e distinguir entre causas comuns e causas especiais de variabilidade.

As causas comuns são originárias da variabilidade natural do processo, sendo, portanto, consideradas aleatórias, sinalizando que o processo está sob controle estatístico. Já as causas especiais são originárias da formação de padrões, sinalizando que algo de especial está influenciando o processo, sendo, portanto necessário identificá-la e removê-la para que toda a produção não seja afetada. Quando causas especiais aparecem, diz-se que o processo está fora de controle estatístico. 
Segundo Montgomery (1997), um processo só está sob controle estatístico quando as causas especiais de variação são eliminadas do processo, e os pontos plotados do gráfico de controle permanecem dentro dos limites de controle.

Segundo Vieira (1999), para planejar um gráfico de controle, é preciso estabelecer o tamanho da amostra e a freqüência da amostragem. Não existem fórmulas para determinar esses valores, mas em geral, é possível escolher uma das duas estratégias:

a) tomar amostras pequenas e freqüentes;

b) tomar amostras grandes e pouco freqüentes.

Os gráficos de controle em estudo são os gráficos da média (X-barra), também chamados de gráficos de controle por variáveis, utilizados para controlar a dispersão do processo. Sua finalidade consiste na melhora da qualidade utilizada no desenvolvimento dos produtos e na capacidade do processo, bem como no auxílio para a tomada de decisão com relação à especificação do produto e ao processo de produção.

\subsection{Construção dos gráficos de controle $x$-barra $(\bar{X})$}

Para a construção dos gráficos de controle X-barra, é preciso primeiramente fazer-se a escolha da variável a ser analisada, cuja característica de qualidade deve ser mensurável. A seguir, define-se também o método de amostragem, se instantâneo ou periódico, e o tamanho da amostra a ser utilizada. Na seqüência, efetua-se a coleta dos dados e determina-se o valor central e os limites de controle, usando a seguinte fórmula:

$$
\overline{\bar{X}}=\frac{\sum_{i=1}^{g} \overline{X_{i}}}{g} \quad \text { e } \quad \bar{s}=\frac{\sum_{i=1}^{g} S_{i}}{g}
$$

onde:

$\overline{\bar{X}}=$ média das médias dos subgrupos;

$\overline{X_{i}}=$ média do i-ésimo subgrupo;

$\mathrm{g}=$ número de subgrupos;

$\mathrm{S}_{\mathrm{i}}=$ desvio padrão do i-ésimo subgrupo;

$\bar{S}=$ média dos desvios padrões dos subgrupos.

Os limites do controle são calculados da seguinte forma:

$$
\begin{aligned}
& \operatorname{LSC} \overline{\bar{x}}=\overline{\bar{X}}+A_{3} \bar{s} \\
& L I C \bar{x}=\overline{\bar{X}}-A_{3} \bar{S}
\end{aligned}
$$


sendo:

$L S C=$ limite superior de controle;

$L I C=$ limite inferior de controle;

$A_{3}=$ fator que varia com o tamanho do subgrupo para o cálculo dos limites de controle.

Em geral, quando se parte para prática, a maioria dos processos não está sob controle quando esses são analisados pela primeira vez, tendo pontos fora dos limites de controle dos gráficos. Destarte, usa-se a técnica de descarte desses pontos no cálculo dos valores revisados.

Para o cálculo do valor central revisado, tem-se:

$$
\overline{X_{0}}=\overline{\overline{X_{r e v}}}=\frac{\sum_{i=1}^{g} \overline{X_{i}}-\sum_{i=1}^{g} \overline{X_{d}}}{g-g_{d}}
$$

onde:

$\overline{X_{d}}=$ média dos subgrupos descartados;

$g_{d}=$ número de subgrupos descartados.

$$
\begin{aligned}
& S_{0}=\overline{S_{\text {rev }}}=\frac{\sum_{i=1}^{g} S_{i}-\sum_{i=1}^{g_{d}} S_{d}}{g-g_{d}} \\
& \sigma_{0}=\frac{S_{0}}{C_{4}}
\end{aligned}
$$

Sendo:

$\sigma_{0}=$ desvio padrão;

$S_{d}=$ desvios padrões dos subgrupos descartados;

$C_{4}=$ fator para estimativa.

Para os cálculos dos limites de controle revisados, tem-se:

$$
\begin{aligned}
& \operatorname{LSC} \overline{\bar{x}}=\overline{\overline{X_{0}}}+A \sigma_{0} \\
& \operatorname{LIC} \overline{\bar{x}}=\overline{\overline{X_{0}}}-A \sigma_{0}
\end{aligned}
$$

Sendo:

$\mathrm{A}=$ fator para cálculo dos limites de controle.

Geralmente as constantes $\mathrm{A}_{3}$ e $\mathrm{C}_{4}$ são obtidas diretamente de programas computacionais, o que elimina a necessidade de uso de tabelas. 


\section{Aplicação da metodologia}

O metabissulfito de sódio é um produto usado mundialmente como inibidor do oxigênio molecular. Durante o processo de despesca do camarão, prevenções são essenciais e dentre elas o tratamento profilático contra o "black spot" é imprescindível. O termo "black spot" refere-se a ocorrência de uma reação química natural de descoloração do tecido do animal e o surgimento de manchas negras decorrentes de ações enzimáticas pela ausência do sódio, conhecida como melanosi.

Para prevenir o aparecimento de estruturas melanínicas, o camarão deverá ser, imediatamente após a captura, tratado com metabissulfito de sódio, mediante a imersão por um período de 15 a 25 minutos numa solução obtida através da diluição prévia do produto metabissulfito de sódio em água gelada, de modo que a concentração do dióxido de enxofre residual atenda as determinações da Organização Mundial de Saúde.

A quantidade de metabissulfito de sódio resultante das despescas deve ser retirada através de bombas elétricas das caixas de imersão para uma pipa, transportada e colocada em tanques distantes no mínimo $500 \mathrm{~m}$ dos afluentes e canais de abastecimento. A referida solução será submetida a um processo de neutralização que consiste na adição de $0,36 \mathrm{~kg}$ de hidróxido de cálcio ou $0,38 \mathrm{~kg}$ de hidróxido de sódio para cada $1 \mathrm{~kg}$ de metabissulfito de sódio. Após esta etapa, o camarão é levado até a indústria dentro de caixotes numa mistura de gelo/camarão/gelo onde ele vai ser lavado e processado. Um dos papéis do controle de qualidade da indústria é coletar amostra desse camarão desde a despesca, analisando os níveis estabelecidos pela Organização Mundial de Saúde.

Neste item apresenta-se a discussão referente ao estudo, juntamente com a análise exploratória dos dados e a avaliação do processo por meio de gráficos de controle.

\subsection{Análise exploratória}

Os dados analisados representam 401 amostras de camarão coletadas na recepção da indústria, que é o primeiro local por onde o camarão passa após a despesca para ser higienizado e processado, e no próprio processo, etapa onde o camarão é separado por gramaturas e embalado. As análises de metabissulfito $\left(\mathrm{SO}_{2}\right)$ são provenientes dos 22 sub-lotes despescados no mês de novembro de 2003 numa fazenda de exportação de camarão no estado do Rio Grande do Norte. Inicialmente apresenta-se uma estatística descritiva da variável, conforme mostra a Figura 01. 


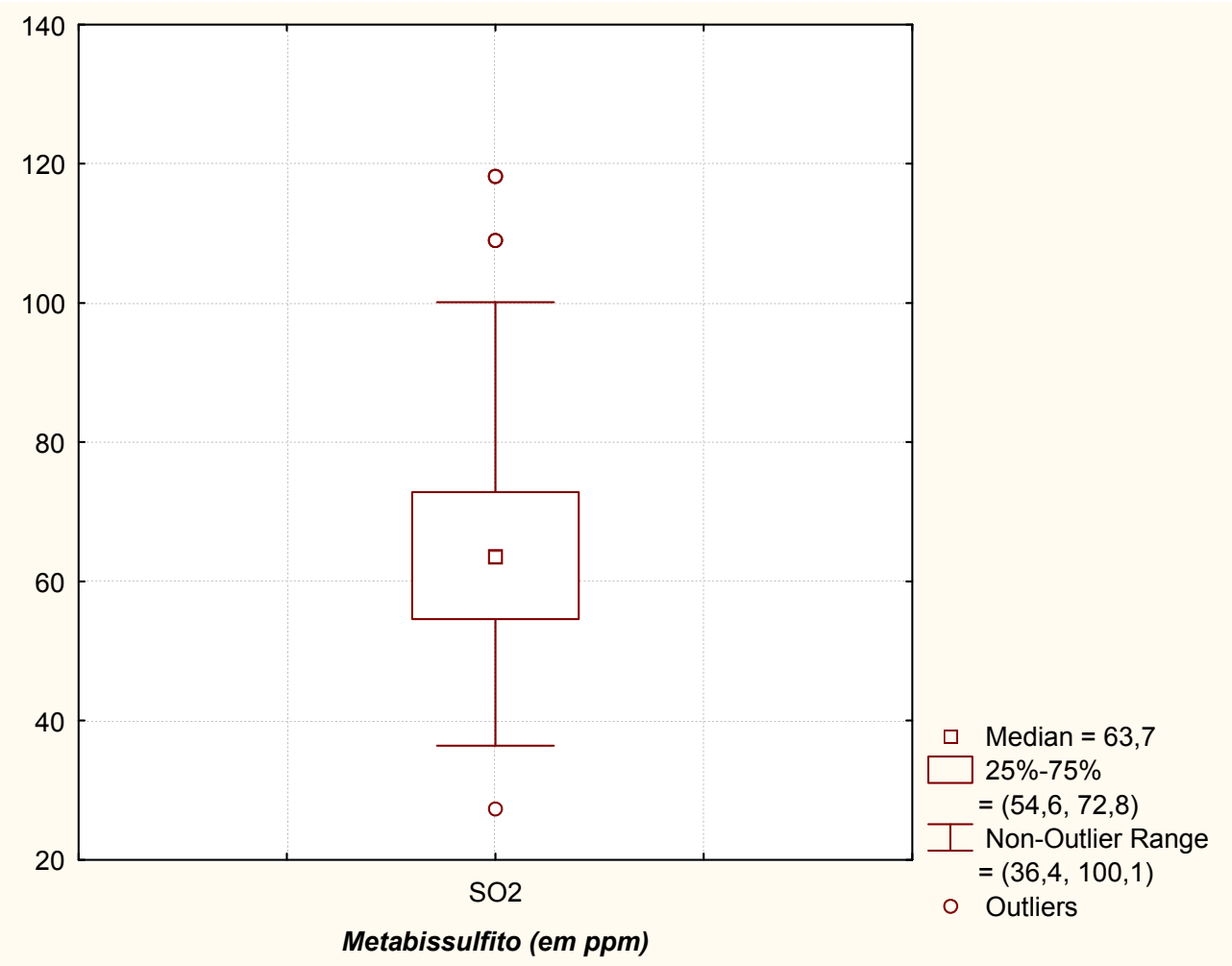

Figura 01 - Gráfico box-plot da variável $\mathrm{SO}_{2}$.

Verifica-se, por meio da Figura 01, que a variável apresenta uma alta variação dos dados, apresentando três outliers. Esta alta variação pode ser comprovada através da Tabela 01, pois se observa um alto desvio padrão, bem como um alto coeficiente de variação. Esse resultado já dá indícios de uma certa variação de metabissulfito entre os lotes analisados.

Tabela 01 - Valores das estatísticas descritivas para a variável metabissulfito $\left(\mathrm{SO}_{2}\right)$.

\begin{tabular}{c|c|c|c|c|c}
\hline Variável & Valor Mínimo & Valor Máximo & Média & Desvio Padrão & $\begin{array}{c}\text { Coeficiente de } \\
\text { variação }\end{array}$ \\
\hline $\mathrm{SO}_{2}(\mathrm{ppm})$ & 27,30 & 118,30 & 63,84 & 15,14 & $23,71 \%$ \\
\hline
\end{tabular}

Fonte: Dados coletados

\subsection{Análise de controle do processo}

O objetivo desta análise é observar o comportamento da variável metabissulfito no processo de despesca e beneficiamento do camarão, que deve ser mantido em níveis estabelecidos pela Organização Mundial de Saúde para garantir uma melhor qualidade do camarão evitando assim, o aparecimento de estruturas melanínicas no mesmo e melhorando sua aparência, que é a maior exigência do consumidor final, principalmente se este produto é destinado para exportação. 
O gráfico a seguir, elaborado no Software Statistica vs 6.0, mostra o comportamento dos 22 sub-lotes analisados. Vale salientar que as 401 amostras são subdivididas entre esses sub-lotes, ou seja, cada sub-lote tem uma média de metabissulfito.

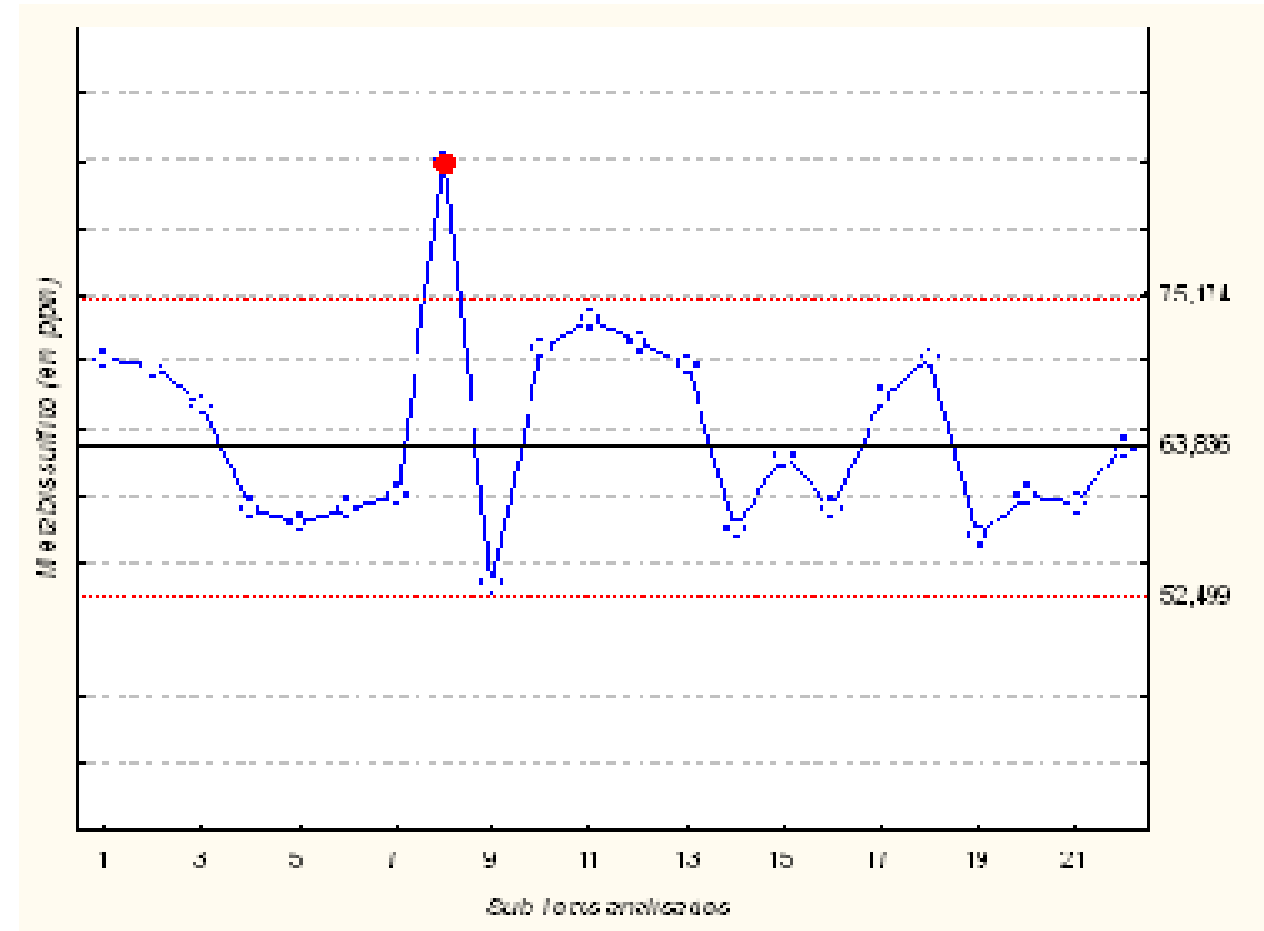

Figura 02 - Gráfico x-barra para a variável $\mathrm{SO}_{2}$

De acordo com o gráfico acima pode-se observar que o $8^{\circ}$ sub-lote encontra-se fora dos limites de controle, sendo necessário algumas mudanças no processo. Uma das possíveis causas que levaram esse sub-lote a apresentar-se fora de controle seria a má utilização do produto, já que, após a captura do camarão existe um período determinado para que este fique imerso na solução de metabissulfito, podendo ter ocorrido uma falha com relação ao tempo de imersão ou uma falha na hora do preparo desta solução. Uma outra possibilidade seria uma falha humana nas análises laboratoriais, em virtude da mesma exigir critérios minuciosos.

Destarte, deve-se nesse momento tomar algumas decisões a respeito desse sub-lote que apresentou uma causa especial de variação, sinalizando que algo de especial está influenciando o processo, deixando-o fora de controle estatístico. Neste caso, depois de identificá-lo, a alternativa é retirar as amostras provenientes desse sub-lote para se calcular os novos limites de controle, podendo ser construídos limites estáveis, os quais servirão para avaliar o processo futuramente.

Depois de retiradas as amostras que faziam parte do $8^{\circ}$ sub-lote, que estava deixando o processo fora de controle estatístico, pôde-se observar que os pontos plotados do gráfíco de controle permaneceram dentro dos limites, levando assim o processo a obter um estado de controle estatístico, como será mostrado na Figura 3. 
A seguir, tem-se o gráfico de controle estatístico revisado, agora com 21 sub-lotes e todos dentro dos limites. Diante disto, deve-se cuidar do processo para que esse estado de controle seja mantido indefinidamente.

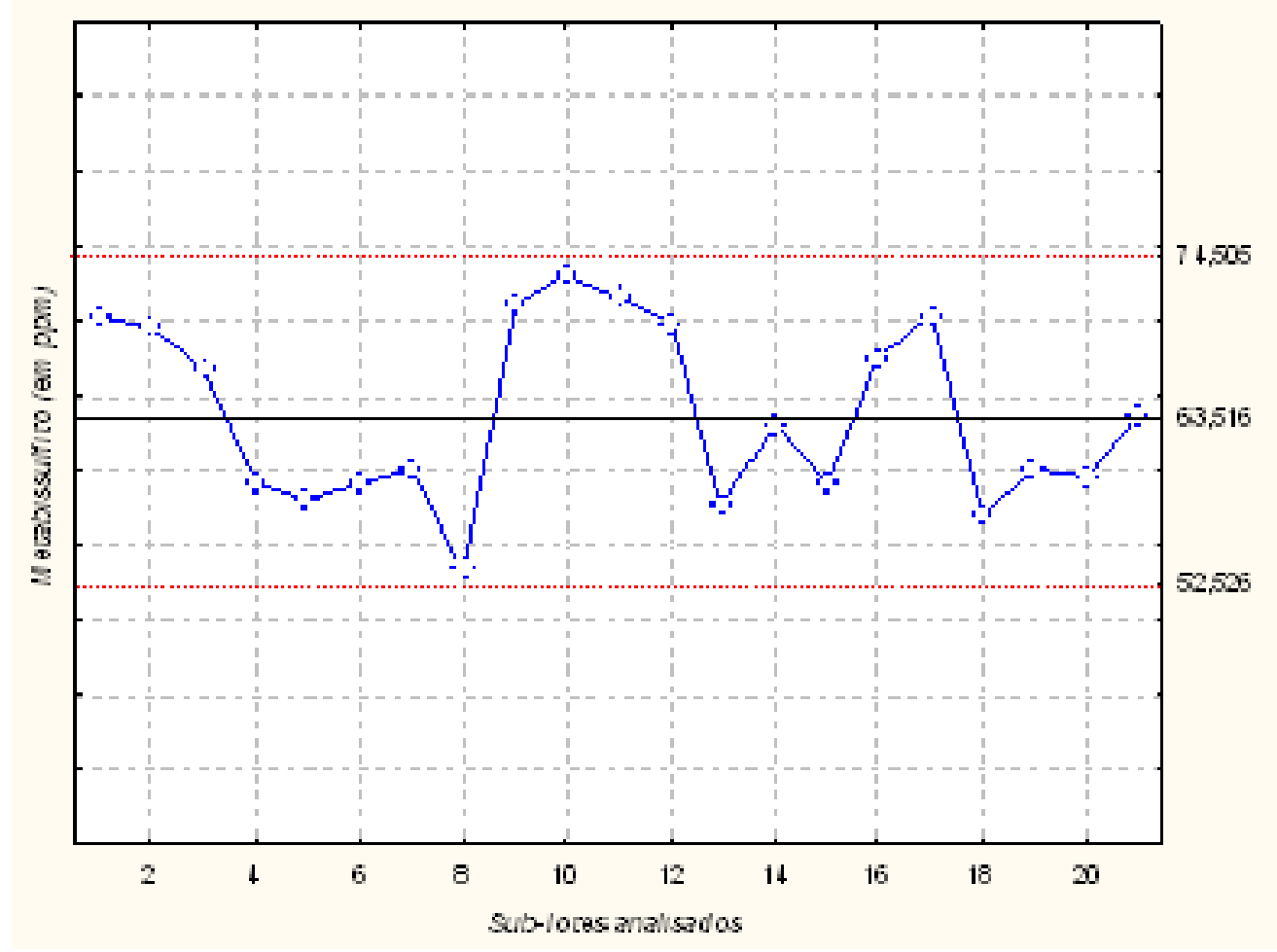

Figura 03 - Gráfico x-barra revisado para a variável $\mathrm{SO}_{2}$

\section{Conclusão}

Este trabalho se propôs a aplicar a técnica estatística Controle Estatístico de Processo, através da utilização da ferramenta Gráfico de Controle, no intuito de monitorar um processo de análises laboratoriais numa indústria de beneficiamento do camarão, em relação à utilização do metabissulfito de sódio, um dos fatores que influenciam na qualidade de conservação dos camarões.

Os dados utilizados para este fim foram coletados durante o mês de novembro de 2003, numa fazenda de exportação de camarão do Rio Grande do Norte, num total de 401 amostras de camarão, onde estas foram subdivididas em 22 sub-lotes para serem feitas às análises do metabissulfito $\left(\mathrm{SO}_{2}\right)$.

De acordo com a análise do controle do processo de beneficiamento do camarão, pôde-se observar inicialmente que este se encontrava fora de controle estatístico, apresentando uma alta variação, apesar de ter apenas um sub-lote fora dos limites de controle. Diante disto, depois de identificado o sub-lote que apresentou uma causa especial de variação, ele foi retirado, para que fossem calculados os novos limites e verificado o novo estado em que se encontrava o processo. 
Logo, observou-se que após esta retirada, os pontos plotados do gráfico de controle permaneceram dentro dos limites, encontrando-se assim, o processo, sob controle estatístico, devendo-se cuidar do processo para que esse estado de controle seja mantido indefinidamente.

Pode-se verificar que a carcinicultura vem apontando como sendo um novo e lucrativo ramo de comércio. Destarte, pesquisas que visem a melhoria de procedimentos, manejo de cultivos e até mesmo, atividades que visem a conservação do meio ambiente, já que tal atividade exercida de maneira incorreta acarretará sérios impactos ao meio ambiente, vem sendo cada vez mais constantes.

Sendo o camarão um produto altamente comerciável tanto nacional como mundialmente, a indústria deste deve manter um alto padrão de qualidade para satisfazer as exigências do mercado, utilizando um sistema de despesca que gere produtos com a qualidade apropriada e aceitação mundial.

\begin{abstract}
This article presents the application of the statistical control of process in an industry of processing of marine shrimp in the state of Rio Grande do Norte, being applied to measure the amount of sodium sulphite, preservative used in the majority of the fishing industries during the process of fishing and processing of shrimp. The variation of amount of sodium sulphite used in the process was measured, and analyzed through the use of control graphs. The results showed that the process did not meet under statistical control, then, after discovery and eliminated the determinable cause of the variation the graph was remade and can be observed that the process met under statistical control.
\end{abstract}

Key words: Quality, Statistical control of process, Sodium sulphite

\title{
6. Referências
}

JURAN, J. M. Juran na liderança pela qualidade. 2. ed. São Paulo: Pioneira, 1993.

SIQUEIRA, Luiz Gustavo Primo. Controle estatístico do processo. São Paulo: Pioneira, 1997.

VIEIRA, Sonia. Estatística para a qualidade: como avaliar com precisão a qualidade em produtos e serviços. Rio de Janeiro: Campus, 1999.

THOMPSON, James R.; KORONACKI, Jacek. Statistical process control for quality improvement. New York: Chapman \& Hall, 1993.

MONTGOMERY, Douglas C. Introduction to statistical quality control. $3^{\text {rd }}$ ed. New York: John Wiley \& Sons, 1997.

JURAN, J. M.; GRYNA, Frank M. Controle da qualidade: métodos estatísticos clássicos aplicados à qualidade. $4^{\text {th }}$ ed. São Paulo: Makron, 1993. v. 6.

CASARIN, Vanusa Andréa; WOLFF, Gilvete Lírio; SOUZA, Adriano Mendonça. Avaliação do processo de transformação da garrafa pet por meio do gráfico X-barra. In: ENCONTRO NACIONAL DE ENGENHARIA DE PRODUÇÃO, 22., Curitiba. Anais... Curitiba: ENEGEP, 2002. 1 CD-ROM. 
METABISSULFITO de sódio: utilização e cuidados ambientais. Jornal do Parceiro - Boletim Informativo Bimestral, Fortaleza, fev. 2003. Disponível em: <http://www.cina.com.br>. Acesso em: 10 maio 2004. 